\title{
Standard model radiative corrections in the pion form factor measurements do not explain the $a_{\mu}$ anomaly
}

\author{
Francisco Campanario, ${ }^{1}$ Henryk Czyż®, ${ }^{2,}{ }^{*}$ Janusz Gluza, ${ }^{2,3}$ Tomasz Jeliński, ${ }^{2}$ Germán Rodrigo, ${ }^{1}$ \\ Szymon Tracz, ${ }^{1}$ and Dmitry Zhuridov ${ }^{2}$ \\ ${ }^{1}$ Instituto de Física Corpuscular, CSIC-Universitat de València, E-46980 Paterna, Valencia, Spain \\ ${ }^{2}$ Institute of Physics, University of Silesia, PL-40-007 Katowice, Poland \\ ${ }^{3}$ Faculty of Science, University of Hradec Králové, CZ-500 03 Hradec Králové, Czech Republic
}

(Received 26 March 2019; published 10 October 2019)

\begin{abstract}
In this paper, we address the question of whether the almost four standard deviations difference between theory and experiment for the muon anomalous magnetic moment $a_{\mu}$ can be explained as a higher-order Standard Model perturbation effect in the pion form factor measurements. This question has, until now, remained open, obscuring the source of discrepancies between the measurements. We calculate the last radiative corrections for the extraction of the pion form factor, which were believed to be potentially substantial enough to explain the data within the Standard Model. We find that the corrections are too small to diminish existing discrepancies in the determination of the pion form factor for different kinematical configurations of low-energy BABAR, BES-III and KLOE experiments. Consequently, they cannot noticeably change the previous predictions for $a_{\mu}$ and decrease the deviations between theory and direct measurements. To solve the above issues, new data and better understanding of low-energy experimental setups are needed, especially as new direct $a_{\mu}$ measurements at Fermilab and J-PARC will provide new insights and substantially shrink the experimental error.
\end{abstract}

DOI: 10.1103/PhysRevD.100.076004

\section{INTRODUCTION}

The anomalous magnetic moment of the muon $a_{\mu} \equiv$ $\left(g_{\mu}-2\right) / 2$ is predicted in the Standard Model (SM) with an accuracy at the level of $0.3 \mathrm{ppm}$ [1-4] while the precision of the direct experimental measurement is of the order of $0.54 \mathrm{ppm} \mathrm{[5].} \mathrm{Remarkably,} \mathrm{the} \mathrm{tension} \mathrm{between}$ the experimental measurement and the SM prediction, $a_{\mu}^{\mathrm{exp}}-a_{\mu}^{\mathrm{SM}}=268(63)(43) \times 10^{-11}$ [4], corresponds to about 3.5 standard deviations. This is one of the largest and longstanding discrepancies between the SM and experiment. The central question is whether the discrepancy is due to unknown new physics effects beyond the SM (new particles and new interactions) or to theoretical and/or experimental errors not completely under control. Concerning the beyond the SM option, there are a few models, which are able to shift the theoretical prediction for $a_{\mu}$ in the direction of the experimental value in selected regions of the parameter space $[6,7]$. However, many commonly used nonstandard

\footnotetext{
* Corresponding author. henryk.czyz@us.edu.pl

Published by the American Physical Society under the terms of the Creative Commons Attribution 4.0 International license. Further distribution of this work must maintain attribution to the author(s) and the published article's title, journal citation, and DOI. Funded by SCOAP ${ }^{3}$.
}

models have problems to accommodate this discrepancy and will have to be modified or rejected, when that harbinger of new physics is confirmed [8]. There are remarkable recent QCD + QED lattice calculations of $a_{\mu}$ [9-12]. However, their accuracy is still far from being competitive with the dispersive/experimental approach [1-4]. Nonetheless, a hybrid lattice/dispersive approach provides currently the most accurate prediction [12], and additional improvements are expected in the pure lattice calculations.

In this paper, we scrutinize possible flaws in the estimation of the theoretical and experimental errors by re-investigating the SM input into data analysis related to pion-photon interactions, including so far missing and potentially relevant radiative corrections.

This study is particularly timely due to the fact that new measurements at Fermilab [13] and J-PARC [14] aim to reduce the experimental error of the direct measurement by a factor of four. Therefore, the theoretical and experimental groups that contribute to the accurate determination of $a_{\mu}$ must point to a similar precision in the near future [15], and also to understand definitely the source of the present discrepancies.

The QED and pure electroweak SM contributions to $a_{\mu}$ are known presently with a satisfactory precision and the biggest errors in the estimation of $a_{\mu}$ arise from the hadronic vacuum polarization [1-3]. One of the main obstacles to reduce the error of the hadronic contribution 
to $a_{\mu}$ is the discrepancy between the experimental extractions of the pion form factor from the cross section of the reaction $e^{+} e^{-} \rightarrow \pi^{+} \pi^{-} \gamma$ by using the initial state radiation (ISR) method [16-22]. The most relevant hadronic contribution (about 70\%) to the determination of $a_{\mu}$ comes from the region of the $\pi^{+} \pi^{-}$invariant mass around the $\rho-\omega$ resonances. The biggest difference [18], between KLOE and BABAR measurements, amounts there to about $2 \%$. It goes even up to $10 \%$ around the $\omega$ resonance region, though that region is very narrow and its contribution to $a_{\mu}$ is smaller. For higher $\pi^{+} \pi^{-}$invariant masses (at $0.9 \mathrm{GeV}$ ) the difference raises to $5 \%$.

A possible source of the discrepancy might be attributed to missing radiative corrections in the event generator(s) used in the experimental analyses since radiative corrections could be different at different energies of the experiments. The Monte Carlo event generator PHOKHARA [23] was used by all the experiments, both for the mode $e^{+} e^{-} \rightarrow \mu^{+} \mu^{-} \gamma$, which serves as a luminosity monitoring process, and the mode $e^{+} e^{-} \rightarrow \pi^{+} \pi^{-} \gamma$, which was used to extract the cross section of the reaction $e^{+} e^{-} \rightarrow \pi^{+} \pi^{-}$, and the corresponding pion form factor. In Ref. [24], the complete QED next-to-leading order (NLO) radiative corrections to the cross section of the reaction $e^{+} e^{-} \rightarrow$ $\mu^{+} \mu^{-} \gamma$ were calculated and implemented in PHOKHARA. It was shown there that the radiative corrections for that process, which were missing in the PHOKHARA event generator at the time of the experimental analyses, were at least one order of magnitude smaller than the discrepancies between experiments. The still missing next-to-nextto leading order initial state radiative corrections were estimated in Ref. [23] to be at most $0.3 \%$. That estimate was later confirmed in Ref. [25]. It is taken as a part of the intrinsic accuracy of the PHOKHARA event generator, which is $0.5 \%$, and is added in the experimental analysis as a part of the systematic error. To improve and control errors in a better way, in what follows, we implement neglected so far corrections in the PHOKHARA event generator and discuss their impact on the determination of the pion form factor for the realistic experimental cuts used by BABAR, BES-III and KLOE.

\section{SETTING}

In Fig. 1, the two new classes of radiative corrections to the $e^{+} e^{-} \rightarrow \pi^{+} \pi^{-} \gamma$ cross section discussed in this work are shown, namely, the final-state radiation corrections (FSRNLO) in diagrams (a) and (b), and the two-virtualphoton (TVP) contributions in (c). These two types of contributions are separately gauge independent, allowing to show separate results for them.

The TVP contributions appear for the first time at NLO and consist of Feynman diagrams with two virtual photons exchanged between the initial-state electron-positron line and the final-state pions, and one extra real photon emitted

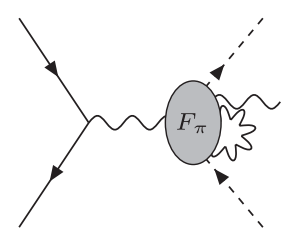

(a)

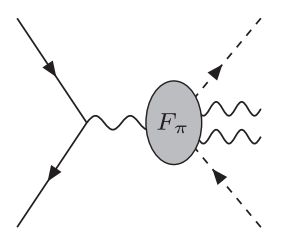

(b)

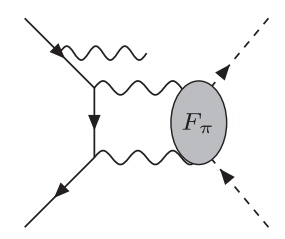

(c)
FIG. 1. Representative final-state radiation (FSRNLO) and two-virtual-photon (TVP) diagrams discussed in the text, which describe the radiative corrections to the $e^{+} e^{-} \rightarrow \pi^{+} \pi^{-} \gamma$ process. In diagram (a) one photon is radiated from one virtual internal line or external line, in diagram (b) two photons are radiated from the final-state pions, in (c) two virtual photons couple both to the final-state pions and initial leptons, and one external photon is emitted either from the initial or the final state.

either from the initial or the final state. They include up to pentagon topologies. At the NLO level, only the interference of these corrections with the Born diagrams contribute to the cross section. These corrections are ultraviolet finite and infrared divergent. To control the numerical accuracy, which is critical in some kinematical regions, the tensor integrals were calculated using the method described in Refs. [26,27] for the 5-point functions with the conventions defined in Ref. [28]. The scalar one-loop integrals were calculated with the QCDLoop library [29] and cross checked against the LoopTools library [30] with quadruple precision. The infrared divergences were regularized in dimensional regularization [31], and were canceled by the appropriate soft photon contributions.

In order to speed up the Monte Carlo event generation, the distributed code works mostly in double precision, and quadruple precision is used only in tensor integrals of TVP contributions to assure the numerical stability. The numerical accuracy of the distributed event generator was also checked against an independent code that was generated with FEYNARTS [32] and FEYNCALC [33]. Both the scalar and the tensor integrals were calculated there in quadruple precision by using the LOOPTOOLS library [30]. A perfect agreement between the two codes was found in phase space points far from the collinear regions. In the collinear regions, where a real photon is emitted along the direction of an initial or final state particle, the numerical accuracy of the distributed code assures 5 significant digits of the result. Gauge independence tests were also performed, as well as tests checking the independence of the result on the slicing parameter separating the phase space of the photon emission into the soft part, where the integral is calculated analytically, and the hard part, where the integral is performed numerically. The gauge independence of the matrix elements holds at the level of $10^{-12}$ relative to the result, while the dependence on the separation parameter yields a numerical precision of $0.02 \%$.

Similar tests were performed for the FSRNLO contributions, which consist of one-loop corrections to the final $\pi^{+} \pi^{-} \gamma$ state and the appropriate two photon real emission. 
These corrections are both ultraviolet and infrared divergent. To cancel the ultraviolet singularities, the renormalization on-shell mass scheme was used. This part of the code is more stable numerically and was kept completely in double precision.

The new open source version 10.0 of the PHOKHARA event generator with complete NLO radiative corrections to the cross section of the reaction $e^{+} e^{-} \rightarrow \pi^{+} \pi^{-} \gamma$ is distributed from the web page [34].

\section{A. Results and discussion}

Having the complete radiative corrections implemented into the event generator PHOKHARA, we can examine how big are these corrections for the event selections used by the $B A B A R$ [16,17], KLOE [18-21] and BES-III [22] collaborations. Their contribution is compared to the predictions of PHOKHARA v9.0 [24], which was used in the experimental analysis of Ref. [22]. In all other analysis [16-21], earlier versions of PHOKHARA were used, which provide identical results as v9.0 for the channel $e^{+} e^{-} \rightarrow \pi^{+} \pi^{-} \gamma$. The most relevant region for the evaluation of the muon anomalous magnetic moment is the pion pair invariant mass $Q^{2}$ in the range $0.6-0.9 \mathrm{GeV}$. This range was also used in the comparison between experiments in the latest KLOE compilation [18]. We will separately discuss the results within and outside this relevant range (RR).

For KLOE, the relative impact of the TVP and FSRNLO radiative corrections, as implemented in PHOKHARA v10.0, is shown in Fig. 2. For the KLOE 2008 event selection [21], they are both below $0.10 \%$ in the RR, and their sum amounts up to $0.18 \%$ at low pion pair invariant masses. At the $\rho$ peak, they are well below $0.05 \%$. Similar results are expected for the event selections used by KLOE in Ref. [19] as the event selection is almost identical to KLOE 2008 [21]. For the KLOE 2010 measurement with a photon tagged in the detector [20], the radiative corrections can be larger, up to $0.5 \%$ in the RR, for both the TVP and the FSRNLO contributions. The sum is also at most $0.5 \%$. Again around the $\rho$ peak they are smaller and amount up to $0.2 \%$. Above $0.9 \mathrm{GeV}$, which is outside the RR, the corrections can be much bigger reaching up to $2.4 \%$. It shows that if one aims to improve the accuracy in this region a dedicated study of the FSR by both experimental and theory groups is necessary.

For the BABAR [16,17] and BES-III [22] event selections, the size of the TVP corrections are shown in Fig. 3. They are below $0.10 \%$ in both cases. The FSRNLO corrections at the energies of these experiments are at least two orders of magnitude smaller than for KLOE, and are thus negligible. The reason for this is that they are proportional to the modulus square of the pion form factor evaluated at the energy of the given experiment and the form factor falls rapidly with the energy.

The radiative corrections involving pions are intrinsically model dependent. Yet, even if we conservatively assume
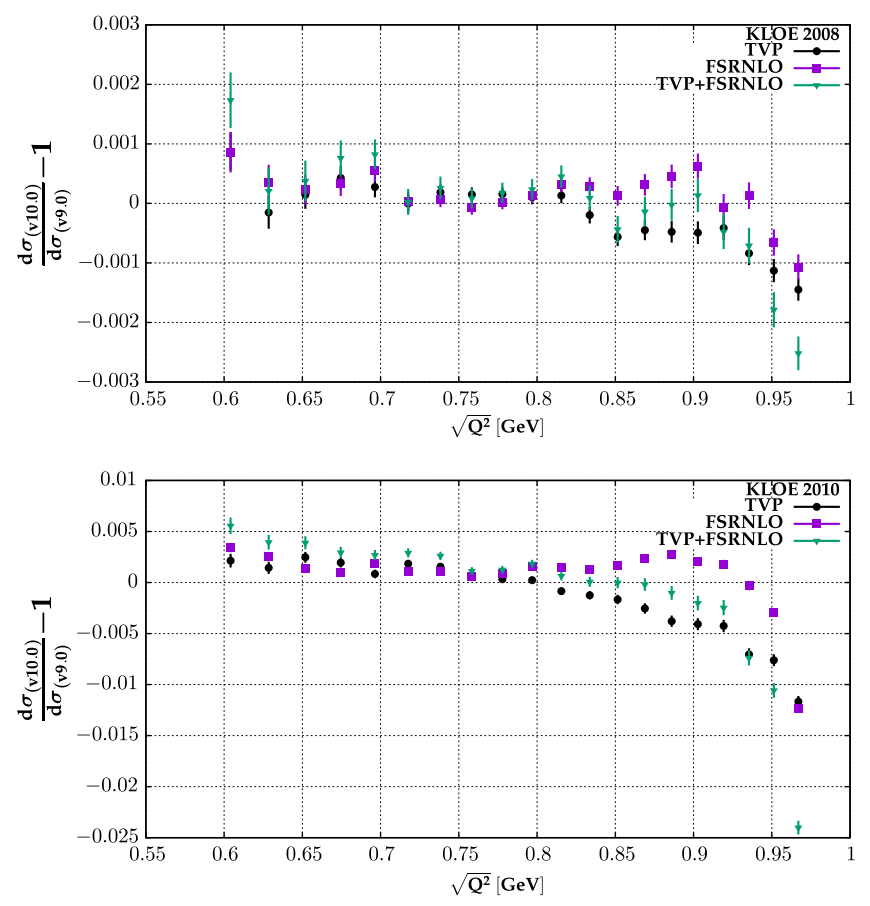

FIG. 2. The size of the TVP and FSRNLO radiative corrections for KLOE [21] (KLOE 2008; photon was not tagged) and [20] (KLOE 2010; tagged photon) event selections as a function of the $\pi^{+} \pi^{-}$invariant mass $Q^{2}$.
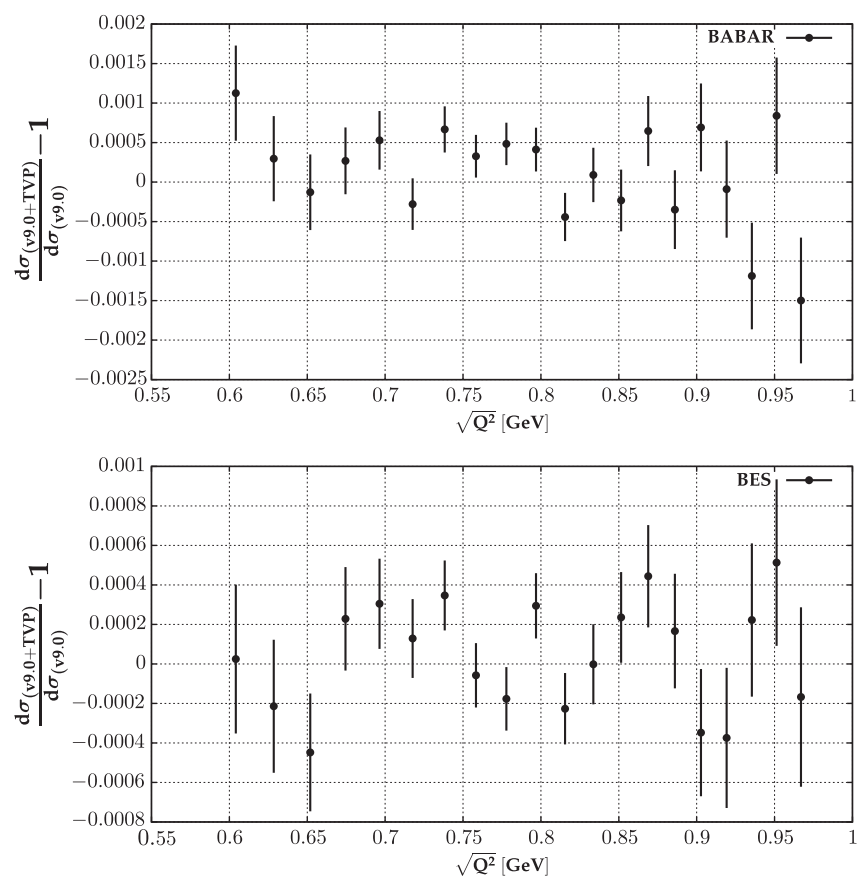

FIG. 3. The size of the TVP radiative corrections for $B A B A R$ [17] and BES-III [22] event selections as a function of the $\pi^{+} \pi^{-}$ invariant mass $Q^{2}$. The FSRNLO contribution is well below $10^{-4}$ and thus TVP + FSRNLO contribution is almost identical with the TVP contribution. 
that the discrepancy is about $50 \%$ of the obtained result, they cannot by any means explain the above mentioned differences between the experimental measurements. The actual accuracy of the presented results is much better than the $50 \%$ mentioned above, as the model used here was well tested experimentally leaving no space for substantial deviations (see Ref. [35] for discussion and further references). A good agreement with the data was found, while additional dedicated tests would be required if a more accurate estimate of the model dependence is needed. This is especially important for KLOE 2010 with the pion pair invariant mass range above $0.9 \mathrm{GeV}$.

\section{SUMMARY AND OUTLOOK}

We conclude that the last set of NLO radiative corrections not considered earlier in the event generator PHOKHARA, which was used by the BABAR, KLOE and BES-III collaborations, cannot be the source of the discrepancies between the different extractions of the pion form factor performed by these experiments. As a consequence, these corrections cannot be the origin of the discrepancy between the experimental measurement and the SM prediction of the muon anomalous magnetic moment $a_{\mu}$ because they are too small. More effort is needed on the experimental side and further, more accurate measurements of the pion form factor are needed to resolve that long standing puzzle, and also to match the expected precision attainable at the next generation of $a_{\mu}$ experiments. With this work, a new version of the event generator PHOKHARA with complete NLO radiative corrections is available for more refined future measurements of the pion form factor.

\section{ACKNOWLEDGMENTS}

The work supported in part by the Polish National Science Centre, Grant No. DEC-2012/07/B/ST2/03867, the German Research Foundation DFG under Contract No. Collaborative Research Center CRC-1044, the Generalitat Valenciana, Spanish Government and ERDF funds from the European Commission (Grants No. PROMETEO/2017/053, FPA2017-84445-P, FPA2017-84543-P and SEV-20140398), and the COST Action PARTICLEFACE (CA16201). F. C. acknowledges financial support by the Spanish Government and Generalitat Valenciana (Grants No. RYC-2014-16061 and SEJI-2017/2017/019). J. G. is supported in part by the Polish National Science Centre, Grant No. DEC-2013/11/B/ST2/04023 and by international mobilities for research activities of the University of Hradec Králové, CZ.02.2.69/0.0/0.0/16_027/0008487.
[1] F. Jegerlehner, The Anomalous Magnetic Moment of the Muon, Springer tracts in modern physics (Springer, Cham, 2017), https://link.springer.com/book/10.1007/978-3-31963577-4.

[2] M. Davier, A. Hoecker, B. Malaescu, and Z. Zhang, Reevaluation of the hadronic vacuum polarisation contributions to the Standard Model predictions of the muon $g-2$ and $\alpha\left(m_{Z}^{2}\right)$ using newest hadronic cross-section data, Eur. Phys. J. C 77, 827 (2017).

[3] A. Keshavarzi, D. Nomura, and T. Teubner, Muon $g-2$ and $\alpha\left(M_{Z}^{2}\right)$ : A new data-based analysis, Phys. Rev. D 97, 114025 (2018).

[4] M. Tanabashi et al., Review of particle physics, Phys. Rev. D 98, 030001 (2018).

[5] G. W. Bennett et al., Final report of the muon E821 anomalous magnetic moment measurement at BNL, Phys. Rev. D 73, 072003 (2006).

[6] M. Bach, D. Stöckinger, H. Stöckinger-Kim, and J.-h. Park, Muon $(g-2)$ in the Standard Model and supersymmetric extensions, EPJ Web Conf. 118, 01034 (2016).

[7] A. Cherchiglia, D. Stöckinger, and H. Stöckinger-Kim, Muon g-2 in the 2HDM: Maximum results and detailed phenomenology, Phys. Rev. D 98, 035001 (2018).
[8] A. Czarnecki and W. J. Marciano, The Muon anomalous magnetic moment: A Harbinger for 'new physics', Phys. Rev. D 64, 013014 (2001).

[9] M. Della Morte, A. Francis, V. Glpers, G. Herdoza, G. von Hippel, H. Horch, B. Jger, H. B. Meyer, A. Nyffeler, and $\mathrm{H}$. Wittig, The hadronic vacuum polarization contribution to the muon $g-2$ from lattice QCD, J. High Energy Phys. 10 (2017) 020.

[10] S. Borsanyi et al., Hadronic Vacuum Polarization Contribution to the Anomalous Magnetic Moments of Leptons from First Principles, Phys. Rev. Lett. 121, 022002 (2018).

[11] B. Chakraborty et al., Strong-Isospin-Breaking Correction to the Muon Anomalous Magnetic Moment from Lattice QCD at the Physical Point, Phys. Rev. Lett. 120, 152001 (2018).

[12] T. Blum, P. A. Boyle, V. Glpers, T. Izubuchi, L. Jin, C. Jung, A. Jttner, C. Lehner, A. Portelli, and J. T. Tsang, Calculation of the Hadronic Vacuum Polarization Contribution to the Muon Anomalous Magnetic Moment, Phys. Rev. Lett. 121, 022003 (2018).

[13] A. Lusiani Muon $g-2$, current experimental status and future prospects, Acta Phys. Pol. B 49, 1247 (2018).

[14] Y. Sato, Muon g-2/EDM experiment at J-PARC, Proc. Sci. KMI2017 (2017) 006. 
[15] Second Plenary Workshop of the Muon g-2 Theory Initiative, 2018, https://wwwth.kph.uni-mainz.de/g-2/.

[16] J. P. Lees et al., Precise measurement of the $e^{+} e^{-} \rightarrow$ $\pi^{+} \pi^{-}(\gamma)$ cross section with the initial-state radiation method at BABAR, Phys. Rev. D 86, 032013 (2012).

[17] B. Aubert et al., Precise Measurement of the $e^{+} e^{-} \rightarrow$ $\pi^{+} \pi^{-}(\gamma)$ Cross Section with the Initial State Radiation Method at BABAR, Phys. Rev. Lett. 103, 231801 (2009).

[18] A. Anastasi et al., Combination of KLOE $\sigma\left(e^{+} e^{-} \rightarrow\right.$ $\left.\pi^{+} \pi^{-} \gamma(\gamma)\right)$ measurements and determination of $a_{\mu}^{\pi^{+} \pi^{-}}$in the energy range $0.10<s<0.95 \mathrm{GeV}^{2}$, J. High Energy Phys. 03 (2018) 173.

[19] D. Babusci et al., Precision measurement of $\sigma\left(e^{+} e^{-} \rightarrow\right.$ $\left.\pi^{+} \pi^{-} \gamma\right) / \sigma\left(e^{+} e^{-} \rightarrow \mu^{+} \mu^{-} \gamma\right)$ and determination of the $\pi^{+} \pi^{-}$ contribution to the muon anomaly with the KLOE detector, Phys. Lett. B 720, 336 (2013).

[20] F. Ambrosino et al., Measurement of $\sigma\left(e^{+} e^{-} \rightarrow \pi^{+} \pi^{-}\right)$ from threshold to $0.85 \mathrm{GeV}^{2}$ using Initial State Radiation with the KLOE detector, Phys. Lett. B 700, 102 (2011).

[21] F. Ambrosino et al., Measurement of $\sigma\left(e^{+} e^{-} \rightarrow \pi^{+} \pi^{-} \gamma(\gamma)\right)$ and the dipion contribution to the muon anomaly with the KLOE detector, Phys. Lett. B 670, 285 (2009).

[22] M. Ablikim et al., Measurement of the $e^{+} e \rightarrow \pi^{+} \pi$ cross section between 600 and $900 \mathrm{MeV}$ using initial state radiation, Phys. Lett. B 753, 629 (2016).

[23] G. Rodrigo, H. Czyż, J. H. Kühn, and M. Szopa, Radiative return at NLO and the measurement of the hadronic crosssection in electron positron annihilation, Eur. Phys. J. C 24, 71 (2002).

[24] F. Campanario, H. Czyż, J. Gluza, M. Gunia, T. Riemann, G. Rodrigo, and V. Yundin, Complete QED NLO contributions to the reaction $e^{+} e^{-} \rightarrow \mu^{+} \mu^{-} \gamma$ and their implementation in the event generator PHOKHARA, J. High Energy Phys. 02 (2014) 114.

[25] S. Jadach, Studies of mu-pair and pi-pair production at the electron-positron low energy colliders, Acta Phys. Pol. B 36, 2387 (2005).

[26] A. Denner and S. Dittmaier, Reduction schemes for one-loop tensor integrals, Nucl. Phys. B734, 62 (2006).

[27] T. Binoth, J. P. Guillet, G. Heinrich, E. Pilon, and C. Schubert, An Algebraic/numerical formalism for one-loop multi-leg amplitudes, J. High Energy Phys. 10 (2005) 015.

[28] F. Campanario, Towards $p p \rightarrow V V j j$ at NLO QCD: Bosonic contributions to triple vector boson production plus jet, J. High Energy Phys. 10 (2011) 070.

[29] R. K. Ellis and G. Zanderighi, Scalar one-loop integrals for QCD, J. High Energy Phys. 02 (2008) 002.

[30] T. Hahn and M. Perez-Victoria, Automatized one loop calculations in four-dimensions and D-dimensions, Comput. Phys. Commun. 118, 153 (1999).

[31] G. 't Hooft and M. J. G. Veltman, Regularization and renormalization of gauge fields, Nucl. Phys. B44, 189 (1972).

[32] T. Hahn, Generating Feynman diagrams and amplitudes with FeynArts 3, Comput. Phys. Commun. 140, 418 (2001).

[33] R. Mertig, M. Bohm, and A. Denner, FEYN CALC: Computer algebraic calculation of Feynman amplitudes, Comput. Phys. Commun. 64, 345 (1991).

[34] Monte Carlo event generator PHOKHARA, http://ific.uv.es/ $\sim$ rodrigo/phokhara/.

[35] S. Actis et al., Quest for precision in hadronic cross sections at low energy: Monte Carlo tools vs. experimental data, Eur. Phys. J. C 66, 585 (2010). 